\title{
Delay performance of cognitive radio networks for point-to-point and point-to-multipoint communications
}

\author{
Hung Tran*, Trung Q Duong and Hans-Jürgen Zepernick
}

\begin{abstract}
In this paper, we analyze the packet transmission time in spectrum sharing systems where a secondary user (SU) simultaneously accesses the spectrum licensed to primary users (PUs). In particular, under the assumption of an independent identical distributed Rayleigh block fading channel, we investigate the effect of the peak interference power constraint imposed by multiple PUs on the packet transmission time of the SU. Utilizing the concept of timeout, exact closed-form expressions of outage probability and average packet transmission time of the SU are derived. In addition, employing the characteristics of the M/G/1 queuing model, the impact of the number of PUs and their peak interference power constraint on the stable transmission condition and the average waiting time of packets at the SU are examined. Moreover, we then extend the analysis for point-to-point to point-to-multipoint communications allowing for multiple SUs and derive the related closed-form expressions for outage probability and successful transmission probability for the best channel condition. Numerical results are provided to corroborate our theoretical results and to illustrate applications of the derived closed-form expressions for performance evaluation of cognitive radio networks.
\end{abstract}

Keywords: cognitive radio networks; spectrum sharing; outage probability; packet transmission time; queueing analysis.

\section{Introduction}

Radio spectrum is one of the most precious and limited resources in wireless communications. It has become scarce due to the rapid growth of a variety of mobile devices and the emerging of many new mobile services. However, recent measurement campaigns conducted by the Federal Communications Commission in the United States have revealed that vast portions of the allocated spectrum are heavily under-utilized [1]. Clearly, the scarcity of the spectrum is due to its inefficient usage rather than a shortage of spectrum resources. As a consequence, the spectrum utilization problem has become more crucial and has stimulated new research such as extensive work on cognitive radio networks (CRN) [2]. In CRNs, there are two types of users who are referred to as primary user (PU) and secondary user (SU). The PU licenses the spectrum while a

${ }^{*}$ Correspondence: hung.tran@bth.se

Radio Communications Group, Blekinge Institute of Technology, SE-371 79 Karlskrona, Sweden
SU may access the spectrum owned by the PU provided that it does not compromise the quality of service (QoS) delivered to the PU. Therefore, a major challenge with the design of CRNs is to maintain the desirable QoS at the PU while offering a sufficiently high transmission rate to the SUs.

Recently, the spectrum sharing approach is considered as a promising solution to utilize the licensed radio frequency. Particularly, the SU and the PU can transmit simultaneously as long as the interference caused by the SU to the PU is lower than a predefined threshold. In [3], considering different fading channels, the ergodic capacity of the spectrum sharing system is investigated for either peak interference power constraint or average received interference power at the PU-Rx. This work has revealed that if the link from the secondary transmitter (SU-Tx) to the primary receiver (PU-Rx) resides in a deep fade, the power of the SU-Tx can be increased to improve the link to the SU-Rx without compromising the peak interference

\section{照 Springer}

(C) 2012 Tran et al.; licensee Springer. This is an Open Access article distributed under the terms of the Creative Commons Attribution License (http://creativecommons.org/licenses/by/2.0), which permits unrestricted use, distribution, and reproduction in any medium, provided the original work is properly cited. 
power constraint. Later, the fundamental capacity limits with imperfect channel knowledge have been studied in $[4,5]$. In [6], the authors have considered a new sophisticated approach for spectrum sharing systems where the impact of channel knowledge on the performance of a secondary user has been studied. The results show that the channel knowledge of the PU-Tx $\rightarrow$ PU-Rx link is important to mitigate the interference from the SU-Tx $\rightarrow$ PU-Rx link while the channel knowledge of the SU-Tx $\rightarrow$ PU-Rx link has little impact on the SU capacity. In [7], different notions of capacity are investigated for the Rayleigh fading channel subject to both the peak and average interference power constraints. Especially, the ergodic capacity and outage capacity which are considered suitable for delay-insensitive and delay-sensitive applications are studied. In [8-10], the novel concept of effective capacity has been introduced to investigate the QoS requirements such as delay constraint in wireless communication systems. In particular, the effective capacity is defined as the maximum constant arrival rate that can be provided by the channel while the delay constraint of the spectrum sharing system is satisfied [9]. The results in [9] have also shown that for a given peak and average interference power constraint at the PU-Rx, the maximal effective capacity is achieved under the optimal power control policy. In relation to the delay constraint in the spectrum sharing system, in [11, 12], we have used another approach, which is based on the packet transmission time to investigate the performance of CRN. These results have revealed the impact of the peak interference power constraint on the delay of packets for different types of fading channels. However, we analyzed the spectrum sharing system with peak interference power constraint only for a single PU.

In this paper, we therefore extend our previous work [11] to consider the more realistic case of a CRN under the peak interference power constraint in the presence of multiple PUs. Specifically, we examine the delay performance for two scenarios, point-to-point and point-tomultipoint communications. In the latter scenario, we extend the investigation from multiple PUs to also allow for multiple SUs at the receiving end. We assume that each packet of the SU-Tx has a delay constraint. In order to not cause harmful interference to any surrounding PU-Rx, the SU-Tx needs to adapt its transmit power and commence transmission before the packet delay threshold is reached. Given this setting, in the point-to-point scenario, we derive the probability density function (PDF) and cumulative density function (CDF) for the packet transmission time, outage probability and average transmission time of packets at the SU-Tx. Furthermore, assuming that packet arrivals at the SU-Tx follow a Poisson process, the queueing model for point-to-point scenario can be described as an $M / G / 1$ system in which packet inter-arrival times are exponentially distributed, service time is a general distribution and traffic is processed by a single server. In the point-to-multipoint scenario, also known as multicast, a secondary base station (SBS) transmits a common packet to all SU-Rx while keeping the peak interference power to the surrounding PU-Rx below a given threshold. By applying the obtained PDF and CDF for the point-to-point scenario, a closed-form expression for the outage probability that the SBS cannot transmit the common packet successfully to a number of SU-Rx are obtained. Moreover, a closed-form expression for the probability that the SBS can transmit the common packet successfully to all SU-Rx, i.e. the best channel condition, is also achieved.

The rest of the paper is organized as follows. In Section 2, the system model and assumptions for the point-to-point and point-to-multipoint scenarios are introduced. In Section 3, analytical formulations for the point-to-point scenario such as the PDF and CDF of the packet transmission time, the outage probability, and the moment of packet transmission time is derived. On this basis, queueing theoretical conclusions are drawn. In Section 4, we present the delay performance for the point-to-multipoint scenario. Section 5 provides numerical results and discussions. Finally, conclusions are presented in Section 6.

\section{System model}

In the sequel, we introduce the point-to-point and pointto-multipoint scenarios in the context of a spectrum sharing system where the SU operates in the area of multiple PUs. As for the radio links between the different entities, we assume identical and independent distributed (i.i.d.) Rayleigh block fading channels with unit-mean in the presence of additive white Gaussian noise (AWGN). The additive noises at both SU-Rx and PU-Rx constitute independent circular symmetric complex Gaussian random variables with zero-mean and variance $N_{0}$, denoted as $\mathcal{C N}\left(0, N_{0}\right)$. As the $\mathrm{SU}$ and the PUs may transmit simultaneously, the interference caused by the SU to the PUs should not exceed a certain threshold.

\subsection{Point-to-point scenario}

Let us consider point-to-point communications in which an SU-Tx is transmitting packets to an SU-Rx while a number $M$ of PU-Rx are operating on the primary network as shown in Figure 1 . The power gain of the SU-Tx $\rightarrow$ SU-Rx link is denoted by $h_{1}$. Similarly, the interference channel power gain of the SU-Tx $\rightarrow$ PU-Rx $x_{m}$ link is denoted by $g_{m}$, $m=1,2, \ldots, M$. Note that channel state information (CSI) of the secondary system can be provided to the SU-Tx through feedback from the SU-Rx while CSI of the SU-Tx to the PU-Rx can be exchanged using a dedicated common control channel [13]. In our study, we follow the assumption given in $[3,5,14,15]$ that the SU-Tx is close to the PU$\mathrm{Rx}$ but the SU-Rx is far away from the primary transmitters (PU-Tx). Therefore, only the SU-Tx causes interference to the PU-Rx while interference caused by the PU-Tx to the SU-Rx is lumped with the AWGN. 


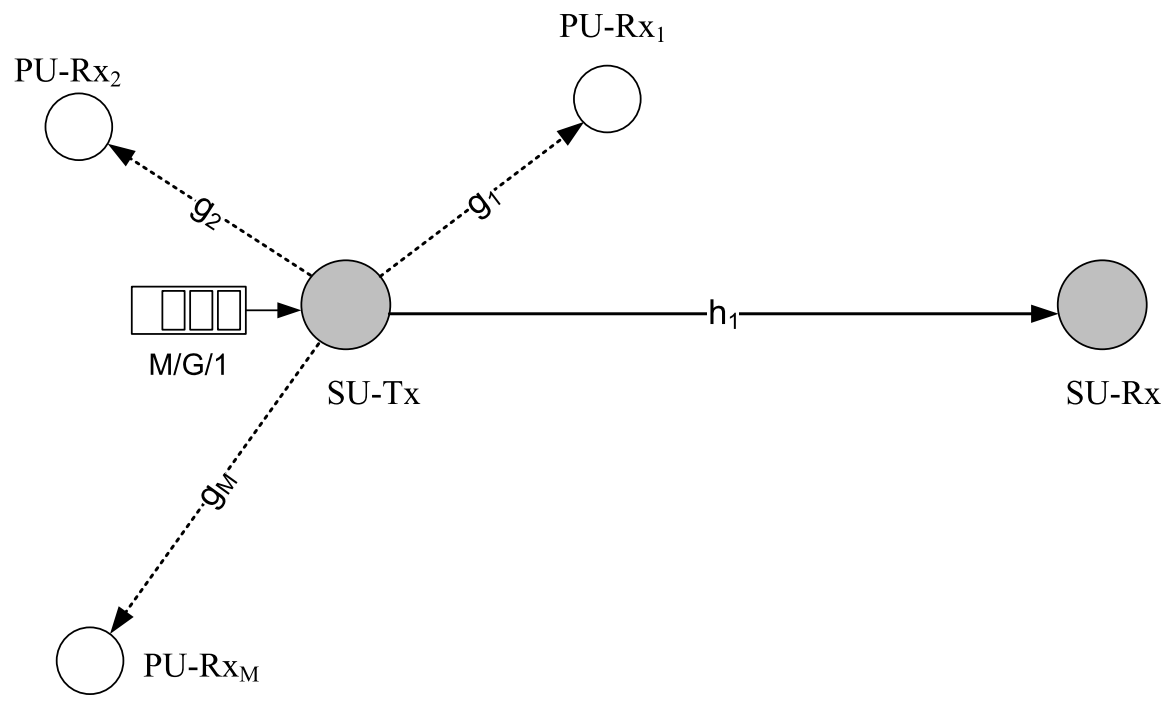

Figure 1 Point-to-point communication of the considered spectrum sharing system with multiple PUs (solid line: communication from SU-Tx to SU-Rx; dashed line: interference from SU-Tx to surrounding PU-Rx).

\subsubsection{Peak interference power constraint}

In order to process the offered traffic, the SU-Tx is equipped with a buffer which stores incoming packets of the same size. The SU-Tx transforms the stored packets into bit streams and adopts its transmission power based on the joined CSI which shall be denoted as $(M+1)$-tuple $\left(g_{1}, g_{2}, \ldots, g_{M} ; h_{1}\right)$. The main objective for the considered spectrum sharing system may be posed as to minimize the transmission time of packets at the SU while not causing harmful interference to the PU-Rx. Following [16], the time taken by an SU-Rx to decode $L$ bits information of a packet can be expressed as

$$
T=\frac{L}{B \log _{2}(1+\gamma)} \triangleq \frac{\widetilde{\mathrm{B}}}{\log _{e}(1+\gamma)}
$$

where $B$ is the system bandwidth, $\widetilde{\mathrm{B}}=L \log _{e}(2) / B$, and $\gamma$ is the signal-to-noise ratio (SNR) at the $\mathrm{SU}-\mathrm{Rx}$ given by

$$
\gamma=\frac{h_{1} P\left(g_{1}, g_{2}, \ldots, g_{M} ; h_{1}\right)}{N_{0}}
$$

In (2), $N_{0}$ represents the noise power spectral density and $P\left(g_{1}, g_{2}, \ldots, g_{M} ; h_{1}\right)$ is the power allocation policy for the SU-Tx corresponding to the joined CSI given as $\left(g_{1}, g_{2}, \ldots\right.$, $\left.g_{M} ; h_{1}\right)$. According to [3], the transmission power of the SU-Tx with respect to $\mathrm{PU}-\mathrm{Rx}_{m}$ should be adjusted to be lower than an allowable level:

$$
g_{m} P\left(g_{1}, g_{2}, \ldots, g_{M} ; h_{1}\right) \leq Q_{\mathrm{pk}}^{m}, \quad m=1,2, \ldots, M
$$

where $Q_{\mathrm{pk}}^{m}$ is the peak interference power that the PU-Rx $x_{m}$ can tolerate without scarifying QoS. Furthermore, let us assume that the tolerable peak interference power is the same for all PU-Rx, i.e. $Q_{\mathrm{pk}}^{m}=Q_{\mathrm{pk}}$ for $m=1,2, \ldots, M$. In order to not cause harmful interference to any PU-Rx in the primary system, the transmission power of the SU-Tx must then satisfy the peak interference power constraint given as

$$
P\left(g_{1}, g_{2}, \ldots, g_{M} ; h_{1}\right) \leq \frac{Q_{\mathrm{pk}}}{\max _{m}\left\{g_{m}\right\}}
$$

\subsubsection{Delay constraint}

As far as the transmission time of packets is concerned, this is clearly non-deterministic due to the fading channel. In the sequel, the transmission of a packet is considered as successful if the packet transmission time is less than a predefined threshold, $t_{\text {out }}$, referred to as timeout. Figure 2 shows an example of a timing diagram of packet transmission for point-to-point communication between SU-Tx and SU-Rx. Recall that the SU-Tx receives packets from higher layers which it will convert into bit streams at the lower layer prior to transmission over the fading channel. Once the SU-Rx has received a sufficient number of bits and decoded the related packets successfully, it will respond with an acknowledgement (ACK) packet that is assumed to be error-free and incurs negligible delay to the SU-Tx. This ACK indicates the SU-Tx that it can eliminate the corresponding packet at the head of the buffer and may continue with transmitting the subsequent packets. In the example shown in Figure 2, the first packet is transmitted unsuccessfully as the SU-Tx does not receive an ACK 


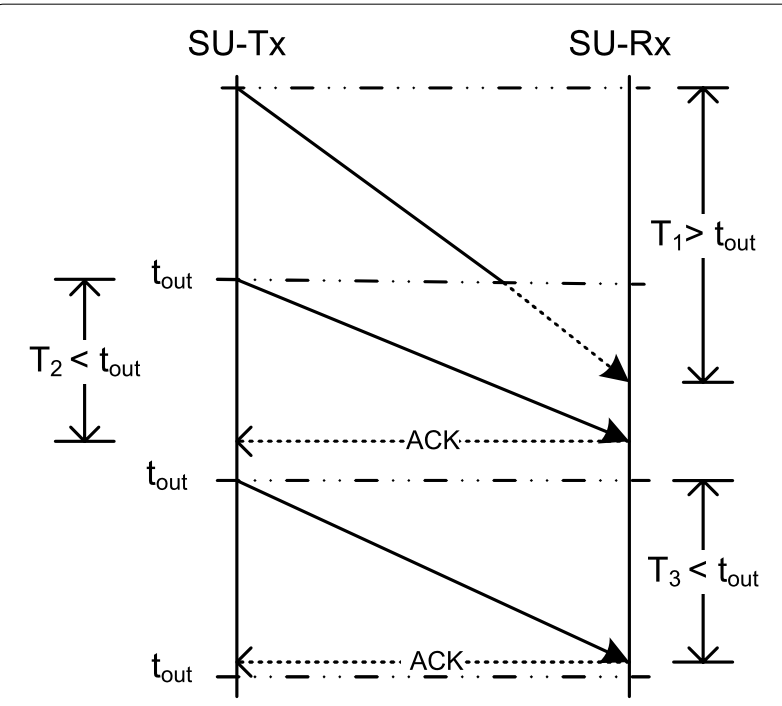

Figure 2 Example of a timing diagram for point-to-point communication between SU-Tx and SU-Rx.

within $t_{\text {out }}$, i.e. $T_{1} \geq t_{\text {out }}$. In this case, the SU-Tx considers the packet as dropped. In contrast, the second and third packet are transmitted successfully as their transmission times are less than the timeout $t_{\text {out }}$, i.e. $T_{2}, T_{3}<t_{\text {out }}$.

\subsubsection{Queuing model for point-to-point communications}

The packets arriving at the SU are stored in a buffer and served in first-in first-out (FIFO) order. Assuming that the packet arrival follows a Poisson process with arrival rate $\lambda$, the considered point-to-point scenario may be modeled as an M/G/1 queueing system [17], [19] with service time given as general distribution and the system being equipped with a single server [20].

From (1) and (2) and the peak interference power constraint (4), we can conclude that the packet transmission time depends on both the channel gain and the peak interference power constraint. Clearly, once the distribution of transmission time is determined, the average waiting time of packets at the SU-Tx can be calculated by applying the Pollaczek-Khinchin's equation [20, Eq. (8.34)] as follows

$$
\mathbb{E}[W]=\mathbb{E}[T]+\mathbb{E}\left[T_{q}\right]
$$

where $\mathbb{E}[W]$ is the total average waiting time of packets at the SU-Tx and $\mathbb{E}\left[T_{q}\right]$ is the average waiting time of packets in the buffer. It is noted that $\mathbb{E}\left[T_{q}\right]$ can be formulated as

$$
\mathbb{E}\left[T_{q}\right]=\frac{\lambda \mathbb{E}\left[T^{2}\right]}{2(1-\rho)}
$$

where $\rho=\lambda \mathbb{E}[T]$ is referred to as channel utilization and $E\left[T^{i}\right], i=1,2$ denotes the first and second moment of packet transmission time, respectively. Furthermore, the following result from queueing theory can be applied for the stability of transmission of the SU.

Stability condition [20] Transmission of the SU-Tx is stable if and only if the average arrival rate $\lambda$ is less than the average transmission rate $\mu$, that is

$$
\lambda<\mu
$$

where average transmission rate is defined by the inverse of the average transmission time as

$$
\mu=\frac{1}{\mathbb{E}[T]}
$$

\subsection{Point-to-multipoint scenario}

In this scenario, we consider a spectrum sharing system as shown in Figure 3 in which a secondary base station (SBS) transmits a common packet to a number $N$ of SU-Rx in its coverage range. This scenario is also known as mobile multicast network in which the base station transmits common information to multiple receivers over broadcast channels $[21,22]$.

\subsubsection{Peak interference power constraint}

In this spectrum sharing scenario, the power allocation problem becomes more complicated as the SBS must not only adjust its power to guarantee successful packet transmission to all SU-Rx in the secondary system but must also limit the interference power caused to the active PU-Rx in the primary system. Clearly, the transmission time of a common packet will vary among the different $\mathrm{SU}-\mathrm{Rx}_{n}$ due to the involved i.i.d. Rayleigh fading channels. Similar to (1), the transmission time of a packet to an SU- $\mathrm{Rx}_{n}$ can be expressed as

$$
T_{n} \triangleq \frac{\widetilde{\mathrm{B}}}{\log _{e}\left(1+\gamma_{n}\right)}, \quad n=1,2, \ldots, N
$$

and $\gamma_{n}$ is the SNR at the $n$th SU-Rx $x_{n}$ which can be formulated as

$$
\begin{aligned}
\gamma_{n} & =\frac{h_{n} P\left(g_{1}, g_{2}, \ldots, g_{M} ; h_{1}, h_{2}, \ldots, h_{N}\right)}{N_{0}}, \\
n & =1,2, \ldots, N
\end{aligned}
$$

where $h_{n}$ is the channel gain from the SBS to the SU- $\mathrm{Rx}_{n}$ while the optimal transmission power $P\left(g_{1}, g_{2}, \ldots, g_{M} ; h_{1}\right.$, $\left.h_{2}, \ldots, h_{N}\right)$ of the SBS is given with respect to the joined CSI denoted as $(M+N)$-tuple $\left(g_{1}, g_{2}, \ldots, g_{M} ; h_{1}, h_{2}, \ldots, h_{N}\right)$. 


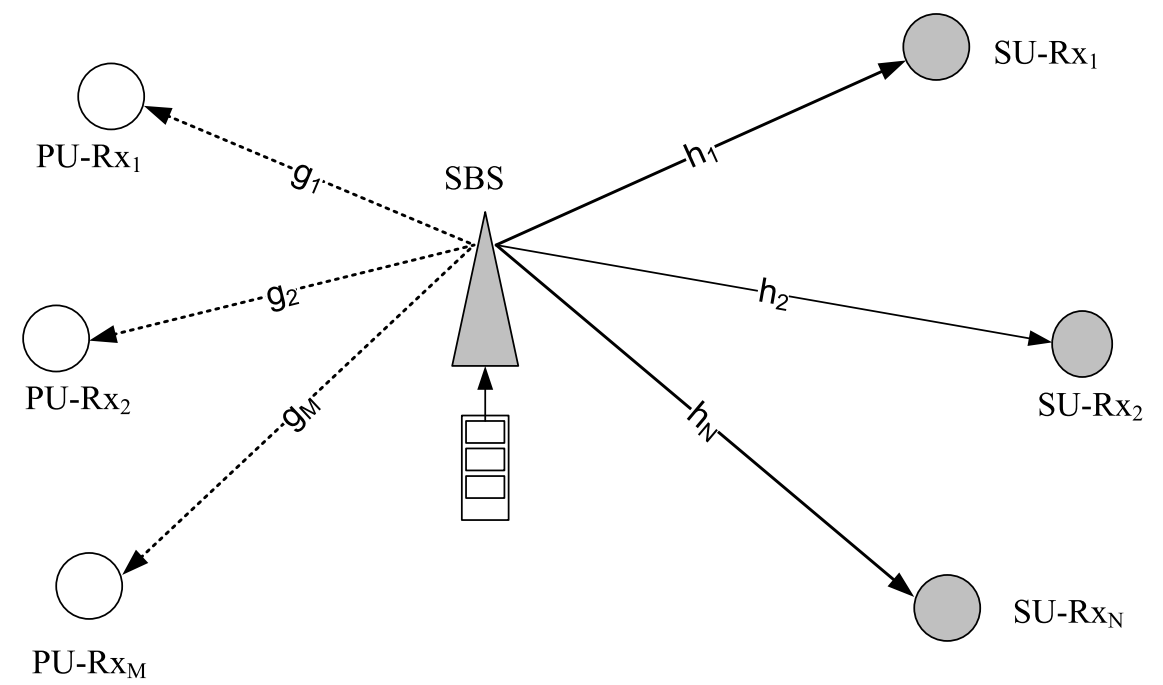

Figure 3 Point-to-multipoint communication of the considered spectrum sharing system with multiple PUs and multiple SUs (solid line: communication from SU-Tx to surrounding SU-Rx; dashed line: interference from SU-Tx to surrounding PU-Rx).

The transmission power policy of the SBS with respect to the PU- $\mathrm{Rx}_{m}$ should then satisfy the following condition:

$$
\begin{aligned}
& g_{m} P\left(g_{1}, g_{2}, \ldots, g_{M} ; h_{1}, h_{2}, \ldots, h_{N}\right) \leq Q_{\mathrm{pk}}^{m}, \\
& \quad m=1,2, \ldots, M
\end{aligned}
$$

Similar to the point-to-point scenario, we assume $Q_{\mathrm{pk}}^{m}=$ $Q_{\mathrm{pk}}$ which leads to the condition for the instantaneous transmission power of SBS as

$$
P\left(g_{1}, g_{2}, \ldots, g_{M} ; h_{1}, h_{2}, \ldots, h_{N}\right) \leq \frac{Q_{\mathrm{pk}}}{\max _{m}\left\{g_{m}\right\}}
$$

\subsubsection{Delay constraint}

In the point-to-multipoint scenario, the SBS tries to broadcast common packets to all SU-Rx in its coverage range. Each common packet has a time-to-live which should be less than $t_{\text {out }}$. If an SU-Rx receives a common packet, it feeds back an ACK to the SBS before $t_{\text {out }}$. This means that the SU-Rx has received the common packet successfully. Otherwise, the SBS implies that the SU-Rx has not received the transmitted packet. Figure 4 shows an example of a timing diagram where the SBS transmits common packets to two SU-Rx. In particular, the SBS transmits the first packet successfully as both transmission times $T_{1,1}$ and $T_{2,1}$ corresponding to SU- $\mathrm{Rx}_{1}$ and SU- $\mathrm{Rx}_{2}$, respectively, are less than the timeout $t_{\text {out }}$. It is noted that $T_{1,1}$ may be different from $T_{2,1}$ due to the different fading channel and spatial separation of SU- $\mathrm{Rx}_{1}$ and $\mathrm{SU}-\mathrm{Rx}_{2}$. In contrast, the second common packet is transmitted unsuccessfully to $\mathrm{SU}-\mathrm{Rx}_{2}$ as the SBS does not receive an ACK from SU$\mathrm{Rx}_{2}$ before timeout $t_{\text {out }}$.

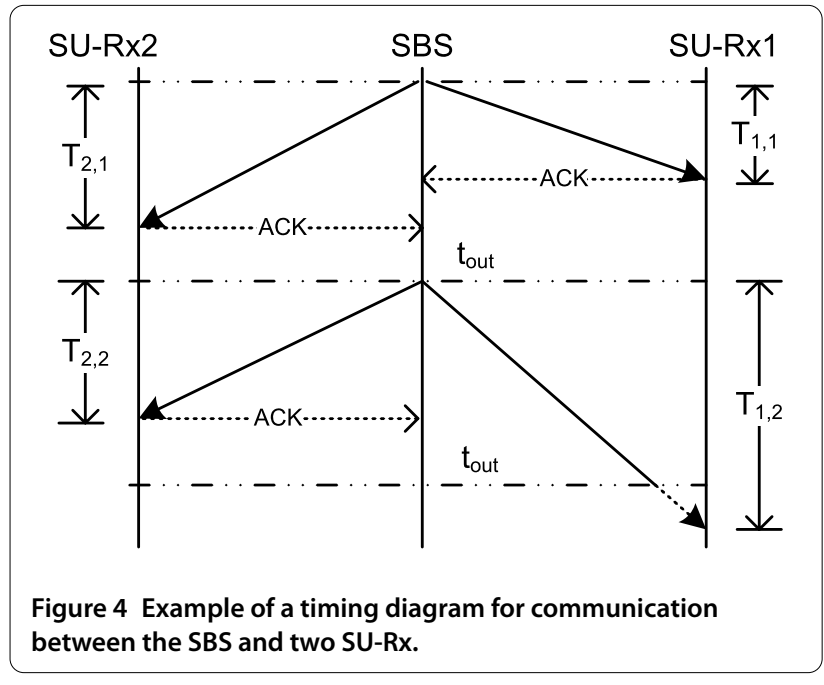

Clearly, if the SBS receives ACKs from all SU-Rx before $t_{\text {out }}$, it can be considered as the best channel condition. On the other hand, the SBS may not transmit the common packet successfully to all SU-Rx due to the fading environment.

\section{Performance analysis for point-to-point communications}

In this section, we derive closed-form expressions for the PDF and CDF of packet transmission time as well as outage probability. Based on these results, we not only quantify the first and second moment of packet transmission time but also investigate the queueing theoretical characteristics of the considered spectrum sharing system. 


\subsection{PDF of packet transmission time}

In this scenario, the SU-Tx wants to transmit with maximum transmission rate in order to reduce dropped packets due to timeout. On the other hand, the SU-Tx not only needs to adjust its transmission power in response to changes of the transmission environment but also guarantee the QoS of any PU-Rx around. Given perfect CSI, the maximum instantaneous transmission power of the SU-Tx in (4) can be expressed with equality as

$$
P\left(g_{1}, g_{2} \ldots, g_{M} ; h_{1}\right)=\frac{Q_{\mathrm{pk}}}{\max _{m}\left\{g_{m}\right\}}
$$

By substituting (13) into (2), we can rewrite (1) as

$$
T=\frac{\widetilde{\mathrm{B}}}{\log _{e}\left(1+\left(h_{1} / \max _{m}\left\{g_{m}\right\}\right)\left(Q_{\mathrm{pk}} / N_{0}\right)\right)}
$$

It is easy to see that the packet transmission time, $T$, now turns out to be a function of multiple random variables, i.e. $h_{1}, g_{m}, m=1,2, \ldots, M$. Therefore, in order to investigate the delay performance, we need to derive the PDF of $T$ in the sequel.

Let us start with the CDF of $g_{0}=\max _{m}\left\{g_{m}\right\}$ where $g_{m}$ is the channel gain. Because the channel coefficients undergo Rayleigh fading, the channel gain, $g_{m}$, is a random variable distributed following an exponential distribution with unit-mean, given by

$$
F_{g_{m}}(y)=1-e^{-y}
$$

Using order statistics, we can easily obtain the CDF and PDF of $g_{0}$, respectively, as follows:

$$
\begin{aligned}
& F_{g_{0}}(y)=\left(1-e^{-y}\right)^{M} \\
& f_{g_{0}}(y)=M e^{-y}\left(1-e^{-y}\right)^{M-1}
\end{aligned}
$$

For convenient derivation, let us denote $Z=h_{1} / g_{0}$. The PDF of $Z$ can be obtained by applying the method presented in [23] as

$$
f_{Z}(z)=\sum_{m=0}^{M-1}\left(\begin{array}{c}
M-1 \\
m
\end{array}\right) \frac{(-1)^{m} M}{(1+m+z)^{2}}
$$

On the other hand, the CDF of $T$ can be formulated as

$$
\begin{aligned}
F_{T}(x)= & \operatorname{Pr}\{T<x\}=1-\operatorname{Pr}\left\{Z<\left(e^{B / x}-1\right) \frac{N_{0}}{Q_{\mathrm{pk}}}\right\} \\
= & 1-\sum_{m=0}^{M-1}\left(\begin{array}{c}
M-1 \\
m
\end{array}\right) \\
& \times \frac{(-1)^{m}\left(e^{B / x}-1\right) M}{(1+m)\left(e^{B / x}+m Q_{\mathrm{pk}} / N_{0}+G\right)}
\end{aligned}
$$

and the PDF of $T$ can be derived by differentiating (19) with respect to $x$ as

$$
\begin{aligned}
f_{T}(x)= & \sum_{m=0}^{M-1}\left(\begin{array}{c}
M-1 \\
m
\end{array}\right) \frac{(-1)^{m} \widetilde{\mathrm{B}} M Q_{\mathrm{pk}}}{N_{0}} \\
& \times \frac{\exp (\widetilde{\mathrm{B}} / x)}{\left(\exp (\widetilde{\mathrm{B}} / x)+m Q_{\mathrm{pk}} / N_{0}+G\right)^{2}}, \quad x \geq 0
\end{aligned}
$$

where $G=\frac{Q_{\mathrm{pk}}}{N_{0}}-1$ is introduced for brevity. It is noted that (20) exactly leads to the PDF of [11, Eq. (10)] for the peak interference power constraint of a single PU-Rx by setting $K=1$.

In the subsequent sections, the important result in (20) will be used to investigate the outage probability, the average transmission time and the average waiting time of packets.

\subsection{Outage probability}

Given the channel conditions and the peak interference power constraint, the outage probability $P_{\text {out }}$ is defined as the probability that the packet transmission time $T$ exceeds the interval $t_{\text {out }}$ :

$$
P_{\text {out }}=\operatorname{Pr}\left(T \geq t_{\text {out }}\right)
$$

From (19), we can easily obtain the closed-form expression for the outage probability as

$$
\begin{aligned}
P_{\text {out }}= & 1-\operatorname{Pr}\left(T<t_{\text {out }}\right)=1-F_{T}\left(t_{\text {out }}\right) \\
= & \sum_{m=0}^{M-1}\left(\begin{array}{c}
M-1 \\
m
\end{array}\right) \\
& \times \frac{(-1)^{m} M}{1+m} \frac{\exp \left(\widetilde{\mathrm{B}} / t_{\text {out }}\right)-1}{\exp \left(\widetilde{\mathrm{B}} / t_{\text {out }}\right)+m Q_{\mathrm{pk}} / N_{0}+G}
\end{aligned}
$$

On the other hand, let $T_{s u c}$ denote the transmission time of a packet given that it is not dropped, i.e.,

$$
T_{\text {suc }}=\left\{T \mid T<t_{\text {out }}\right\}
$$


Accordingly, applying Bayes' rule, the probability that the event $T_{\text {suc }}$ takes place can be expressed as

$$
P\left\{T \mid T<t_{\text {out }}\right\}=\frac{P\left\{T, T<t_{\text {out }}\right\}}{P\left\{T<t_{\text {out }}\right\}}=\frac{P\left\{T, T<t_{\text {out }}\right\}}{1-P_{\text {out }}}
$$

Based on (24), we can express the CDF of $T_{\text {suc }}$ as follows:

$$
F_{T_{\text {suc }}}(x)=\frac{1}{1-P_{\text {out }}} \int_{0}^{x} f_{T}(t) d t, \quad 0 \leq x<t_{\text {out }}
$$

and $F_{T_{\text {suc }}}(x)=0$ for $x \geq t_{\text {out }}$. Differentiating both sides of (25) with respect to $x$, the PDF of the packet transmission time without being timed out can be presented as

$$
f_{T_{\text {suc }}}(x)=\frac{d}{d x} F_{T_{\text {suc }}}(x)=\frac{f_{T}(x)}{1-P_{\text {out }}}, \quad 0 \leq x<t_{\text {out }}
$$

and $f_{T_{\text {suc }}}(x)=0$ for $x \geq t_{\text {out }}$. Substituting (20) into (26), the PDF of packet transmission time without being timed out can be obtained as

$$
\begin{aligned}
f_{T_{\text {suc }}}(x)= & \sum_{m=0}^{M-1}\left(\begin{array}{c}
M-1 \\
m
\end{array}\right) \frac{(-1)^{m} \widetilde{\mathrm{B}} M Q_{\mathrm{pk}}}{\left(1-P_{\text {out }}\right) N_{0}} \\
& \times \frac{\exp (\widetilde{\mathrm{B}} / x)}{\left[\exp (\widetilde{\mathrm{B}} / x)+m Q_{\mathrm{pk}} / N_{0}+G\right]^{2}}, \\
& 0 \leq x<t_{\text {out }}
\end{aligned}
$$

while $f_{T_{\text {suc }}}(x)=0$ for $x \geq t_{\text {out }}$. In the following, the PDF $f_{T_{\text {suc }}}(x)$ given in (27) will be used to derive the moment of packet transmission time.

\subsection{Moment of packet transmission time}

Let us recall that a transmitted packet can be received successfully or not due to the fading channel. Therefore, examining average transmission time shall consider both packet transmission time without and with timeout.

Let us start with the average transmission time of packet without timeout as follows

$$
\begin{aligned}
\mathbb{E}\left[T_{\text {suc }}\right]= & \int_{0}^{t_{\text {out }}} x f_{T_{\text {suc }}}(x) d x \\
= & \frac{M Q_{\mathrm{pk}}}{\left(1-P_{\text {out }}\right) N_{0}} \sum_{m=0}^{M-1}\left(\begin{array}{c}
M-1 \\
m
\end{array}\right)(-1)^{m} \\
& \times \int_{0}^{t_{\text {out }}} \frac{\widetilde{\mathrm{B}} \exp (\widetilde{\mathrm{B}} / x)}{x\left[\exp (\widetilde{\mathrm{B}} / x)+m \mathrm{Q}_{\mathrm{pk}} / N_{0}+G\right]^{2}} d x
\end{aligned}
$$

By setting $t=\exp (\widetilde{\mathrm{B}} / x)$ and applying an exchange of variables in the integral of (28), we finally obtain the first mo- ment of packet transmission time without timeout as

$$
\mathbb{E}\left[T_{\text {suc }}\right]=\frac{M Q_{\mathrm{pk}}}{\left(1-P_{\text {out }}\right) N_{0}} \psi_{1}\left(\exp \left(\widetilde{\mathrm{B}} / t_{\text {out }}\right), G\right)
$$

where

$$
\begin{aligned}
\psi_{1}(a, b)= & \sum_{m=0}^{M-1}\left(\begin{array}{c}
M-1 \\
m
\end{array}\right)(-1)^{m} \\
& \times \int_{a}^{\infty} \frac{\widetilde{\mathrm{B}}}{\left(\log _{e} t\right)\left(t+m Q_{\mathrm{pk}} / N_{0}+b\right)^{2}} d t
\end{aligned}
$$

Similarly, we can calculate the second moment of packet transmission time without timeout as follows

$$
\begin{aligned}
\mathbb{E}\left[T_{\text {suc }}^{2}\right]= & \int_{0}^{t_{\text {out }}} x^{2} f_{T_{\text {suc }}}(t) d t \\
= & \frac{M Q_{\mathrm{pk}}}{\left(1-P_{\text {out }}\right) N_{0}} \sum_{m=0}^{M-1}\left(\begin{array}{c}
M-1 \\
m
\end{array}\right)(-1)^{m} \\
& \times \int_{0}^{t_{\text {out }}} \frac{\widetilde{\mathrm{B}} \exp (\widetilde{\mathrm{B}} / x)}{\left[\exp (\widetilde{\mathrm{B}} / x)+m Q_{\mathrm{pk}} / N_{0}+G\right]^{2}} d x
\end{aligned}
$$

Using similar exchange of variables as above for (31), we obtain the second moment of $T_{s u c}$ as

$$
\mathbb{E}\left[T_{\text {suc }}^{2}\right]=\frac{M Q_{\mathrm{pk}}}{\left(1-P_{\text {out }}\right) N_{0}} \psi_{2}\left(\exp \left(\widetilde{\mathrm{B}} / t_{\text {out }}\right), G\right)
$$

where

$$
\begin{aligned}
\psi_{2}(a, b)= & \sum_{m=0}^{M-1}\left(\begin{array}{c}
M-1 \\
m
\end{array}\right)(-1)^{m} \\
& \times \int_{a}^{\infty} \frac{\widetilde{\mathrm{B}}^{2}}{\left(\log _{e}^{2} t\right)\left[t+m Q_{\mathrm{pk}} / N_{0}+b\right]^{2}} d t
\end{aligned}
$$

Finally, by applying the law of total expectation, the first and the second moment of packet transmission time (including dropped packets) can be given by

$$
\mathbb{E}\left[T^{i}\right]=\left(1-P_{\text {out }}\right) \mathbb{E}\left[T_{\text {suc }}^{i}\right]+t_{\text {out }}^{i} P_{\text {out }}, \quad i=1,2
$$

where $P_{\text {out }}$ is given by (22) and $\mathbb{E}\left[T_{\text {suc }}^{i}\right], i=1,2$ can be calculated by (29) and (32), respectively.

\subsection{Queuing theoretical characteristics}

Firstly, the expression for the average waiting time of packets in the buffer of SU-Tx can be obtained by substituting 
(34) with respect to $i=1,2$ into (5) and (6) as

$$
\begin{aligned}
\mathbb{E}[W]= & \frac{N_{0} t_{\text {out }} P_{\text {out }}+M Q_{\mathrm{pk}} \psi_{1}\left(\exp \left(\widetilde{\mathrm{B}} / t_{\text {out }}\right), G\right)}{N_{0}} \\
& +\left[\lambda M Q_{\mathrm{pk}} \psi_{2}\left(\exp \left(\widetilde{\mathrm{B}} / t_{\text {out }}\right), G\right)\right. \\
& \left.+\lambda N_{0} t_{\text {out }}^{2} P_{\text {out }}\right] \\
& /\left[2 \left[N_{0}-\lambda M Q_{\mathrm{pk}} \psi_{1}\left(\exp \left(\widetilde{\mathrm{B}} / t_{\text {out }}\right), G\right)\right.\right. \\
& \left.\left.-\lambda t_{\text {out }} P_{\text {out }}\right]\right]
\end{aligned}
$$

Secondly, the transmission of an SU is stable if and only if the average arrival rate is less than the average transmission rate. Thus, we can make a statement about the stable transmission condition as follows:

Remark Given the channel state information and the peak interference power constraint of $M$ PUs, the transmission of the SU is stable if and only if the average arrival rate of packet, $\lambda$, satisfies the condition

$$
\lambda<\frac{1}{\left(1-P_{\text {out }}\right) \mathbb{E}\left[T_{\text {suc }}\right]+t_{\text {out }} P_{\text {out }}}
$$

The inequality (36) is derived by substituting (34) for $i=1$ into (7).

Finally, by using the Little theorem [20, Eq. (8.2)], the average number of packets waiting in the buffer of the SU-Tx can be formulated as

$$
N=\lambda \mathbb{E}[W]
$$

where $\mathbb{E}[W]$ is given by (35).

\section{Performance analysis for point-to-multipoint communications}

In this section, we consider point-to-multipoint communications, in which both SU and PU links undergo Rayleigh fading. We first derive the exact closed-form expression for the outage probability of the secondary system, and then we consider the probability for the special case that the SBS can transmit the common packet successfully to all SU-Rx in its coverage range.

\subsection{Outage probability}

In the point-to-multipoint scenario, the SBS transmits common packets to SU-Rx in its coverage. Some SU-Rx may not receive the common packets successfully due to fading environment. In order to analyze the performance of this scenario, we will calculate the probability that $k$ out of the total of $N$ SU-Rx cannot receive the common packets successfully, known as outage probability.
Similar to point-to-point communications, the event that the $\mathrm{SU}-\mathrm{Rx}_{n}$ cannot receive a packet successfully is formulated as $T_{n} \geq t_{\text {out }}$ where $T_{n}$ is an i.i.d. random variable distributed following the CDF given by (19). Therefore, the outage probability in this case can be formulated as

$$
\begin{aligned}
P_{\text {out }}^{k}= & \left(\begin{array}{c}
N \\
k
\end{array}\right) \operatorname{Pr}^{k}\left\{T_{n} \geq t_{\text {out }}\right\}\left(1-\operatorname{Pr}\left\{T_{n} \geq t_{\text {out }}\right\}\right)^{N-k} \\
= & \sum_{j=0}^{N-k}\left(\begin{array}{c}
N \\
k
\end{array}\right)\left(\begin{array}{c}
N-k \\
j
\end{array}\right)(-1)^{j}\left[\sum_{m=0}^{M-1}\left(\begin{array}{c}
M-1 \\
m
\end{array}\right)\right. \\
& \left.\times \frac{(-1)^{m} M\left(\exp \left(\widetilde{\mathrm{B}} / t_{\text {out }}\right)-1\right)}{(1+m)\left(\exp \left(\widetilde{\mathrm{B}} / t_{\text {out }}\right)+m \mathrm{Q}_{\mathrm{pk}} / N_{0}+G\right)}\right]^{k+j}
\end{aligned}
$$

where (38) is obtained by using the binomial theorem and the help of (19).

\subsection{Best channel condition}

For point-to-multipoint communications, the SBS may transmit common packets successfully to all SU-Rx if the channel condition is ideal. This is known as the best channel condition which can be expressed as the longest transmission time for one common packet to be less than $t_{\text {out }}$, i.e., $\left\{\max _{n}\left\{T_{n}\right\}<t_{\text {out }}\right\}$. Therefore, the probability that the SBS transmits the common packet to N SU-Rx with the best channel condition can be given as

$$
\begin{aligned}
\operatorname{Pr} & \left\{\max _{n}\left\{T_{n}\right\}<t_{\text {out }}\right\} \\
= & \prod_{n=1}^{N} \operatorname{Pr}\left\{T_{n}<t_{\text {out }}\right\} \\
= & {\left[\sum_{m=0}^{M-1}\left(\begin{array}{c}
M-1 \\
m
\end{array}\right)\right.} \\
& \left.\times \frac{(-1)^{m} M\left(\exp \left(\widetilde{\mathrm{B}} / t_{\text {out }}\right)-1\right)}{(1+m)\left(\exp \left(\widetilde{\mathrm{B}} / t_{\text {out }}\right)+m Q_{\mathrm{pk}} / N_{0}+G\right)}\right]^{N}
\end{aligned}
$$

where (39) can be calculated with the help of (19).

\section{Numerical results}

We first provide numerical results for point-to-point communications. In particular, we study the impact of the peak interference power constraint and the number of PUs on the outage probability, average transmission time and queuing theoretical characteristics of the secondary system. We then discuss results about the outage probability and the probability that the SBS can transmit the common packet successfully under the best channel condition for point-to-multipoint communications. The system parameters are selected following [16] as follows: 


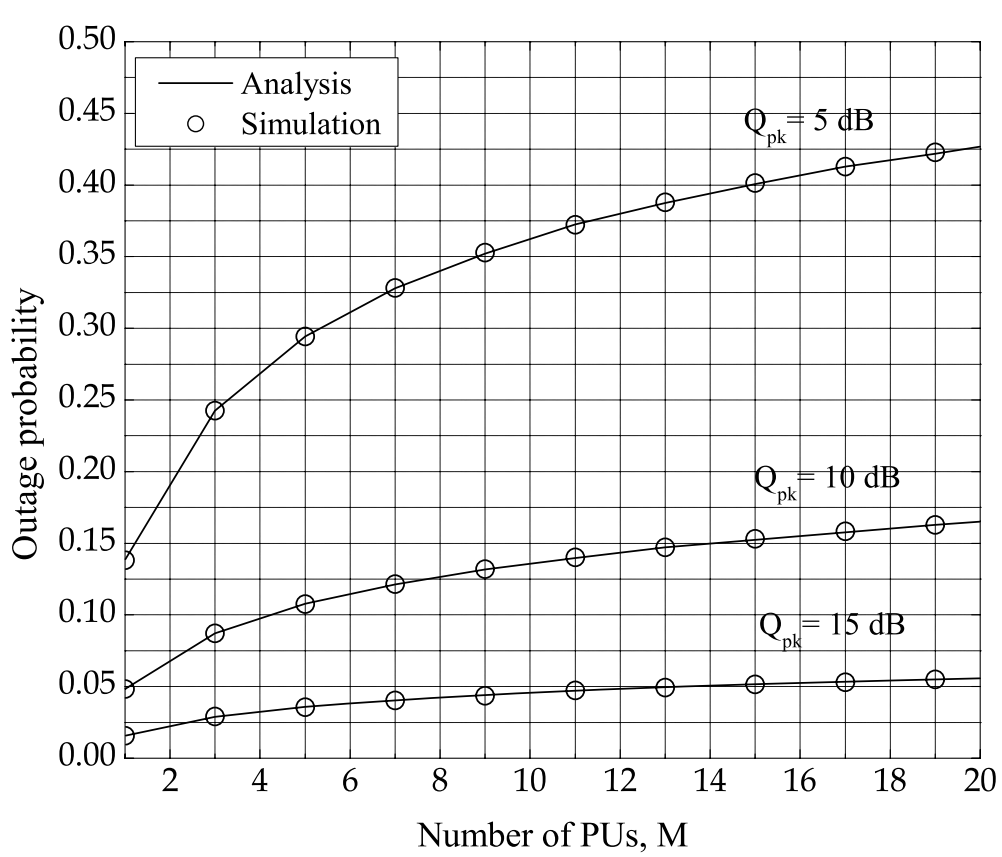

Figure 5 Impact of the number of PUs, $M$, on the outage probability $P_{\text {out }}$ of transmission at the SU-Tx for different values of the peak interference power $Q_{\mathrm{pk}}$.

- System bandwidth: $B=1 \mathrm{MHz}$

- Packet size: $L=4096$ bits (512 bytes)

- Timeout: $t_{\text {out }}=10 \mathrm{~ms}$

- Noise power spectral density: $N_{0}=1 \mathrm{~W} / \mathrm{Hz}$

\subsection{Point-to-point communications}

In the sequel, we focus on the impact of the peak interference power constraint and the number of PUs on the performance of an SU.

\subsubsection{Outage probability}

Figure 5 shows the outage probability as a function of the number of PUs, $M$, for given peak interference power of $Q_{\mathrm{pk}}=5,10,15 \mathrm{~dB}$. As can clearly be observed from the figure, the analysis matches very well with the simulation results in all cases of $Q_{\mathrm{pk}}$. The outage probability increases fast with $M$ if the peak interference power is set to a low value such as $Q_{\mathrm{pk}}=5 \mathrm{~dB}$. On the other hand, the outage probability increases slowly when the peak interference power is high, $Q_{\mathrm{pk}}=10,15 \mathrm{~dB}$ and specifically saturates fast for $Q_{\mathrm{pk}}=15 \mathrm{~dB}$. These results are thought to be due to the fact that an SU-Tx can transmit with relative high transmission power and hence increased transmission rate when the peak interference power $Q_{\mathrm{pk}}$ is large. As a result, the transmission time for the packets can be kept low which in turn reduces the outage probability. On the other hand, for a fixed value of the peak interference power, $Q_{\mathrm{pk}}$, the more PUs operate actively in the primary network, the more constraints are put on the transmission power of an SU-Tx resulting in an increased outage probability (see also (3) and (22)).

\subsubsection{Average transmission time}

Figure 6 depicts the average transmission time of packets at the SU-Tx as a function of the peak interference power, $Q_{\mathrm{pk}}$, for the number of PUs given as $M=1,4,7,10$. Again, analytical and simulation results are in excellent agreement. It can be seen from the figure that the average transmission time for the packets from the SU-Tx decreases as the peak interference power increases. Typically, the average transmission time reduces very fast in the high regime of the peak interference power of about $Q_{\mathrm{pk}} \geq 16.5 \mathrm{~dB}$. This is due to the same reason as discussed above for the outage probability, i.e., an increase of the allowed peak interference power induces a higher transmission rate and hence a decrease of transmission time of packets from the SU-Tx. It should also be noted that the results for the average transmission time matches exactly with our previous results reported in [11, Fig. 3] where we considered the special case of only a single PU being present, i.e., $M=1$. The results shown in Figure 7 enable us to study the impact of the number of PUs on the average transmission time of packets at the SU-Tx. Apparently, the number of PUs has a significant influence on the average transmission time at low values of the peak interference power, say $Q_{\mathrm{pk}}=5 \mathrm{~dB}$, causing it to rapidly increase with 

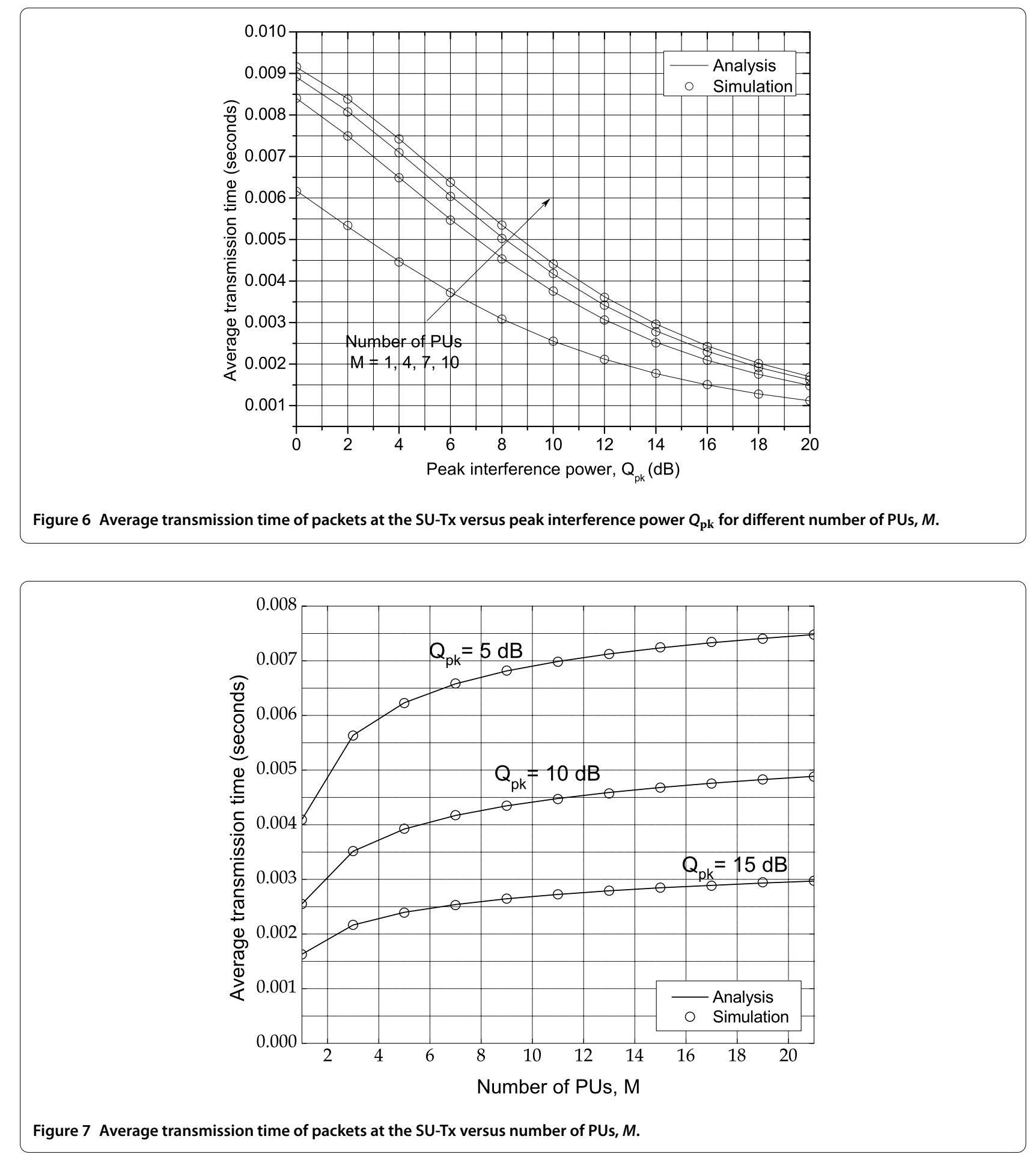

$M$. In contrast, for higher peak interference power such as $Q_{\mathrm{pk}}=10 \mathrm{~dB}$, an increase of the number of PUs increases the average transmission time only slowly and has almost now impact for $Q_{\mathrm{pk}}=15 \mathrm{~dB}$ once $M>4$. These results are consistent with the behavior observed for the outage probability.

\subsubsection{Queuing theoretical results}

In the following, we examine the queuing characteristics of the SU-Tx under the peak interference power constraint (4) with related results shown in Figures 8 and 9. Specifically, we have set the number of PUs to $M=1,3,5$ and observe the average waiting time and channel utiliza- 

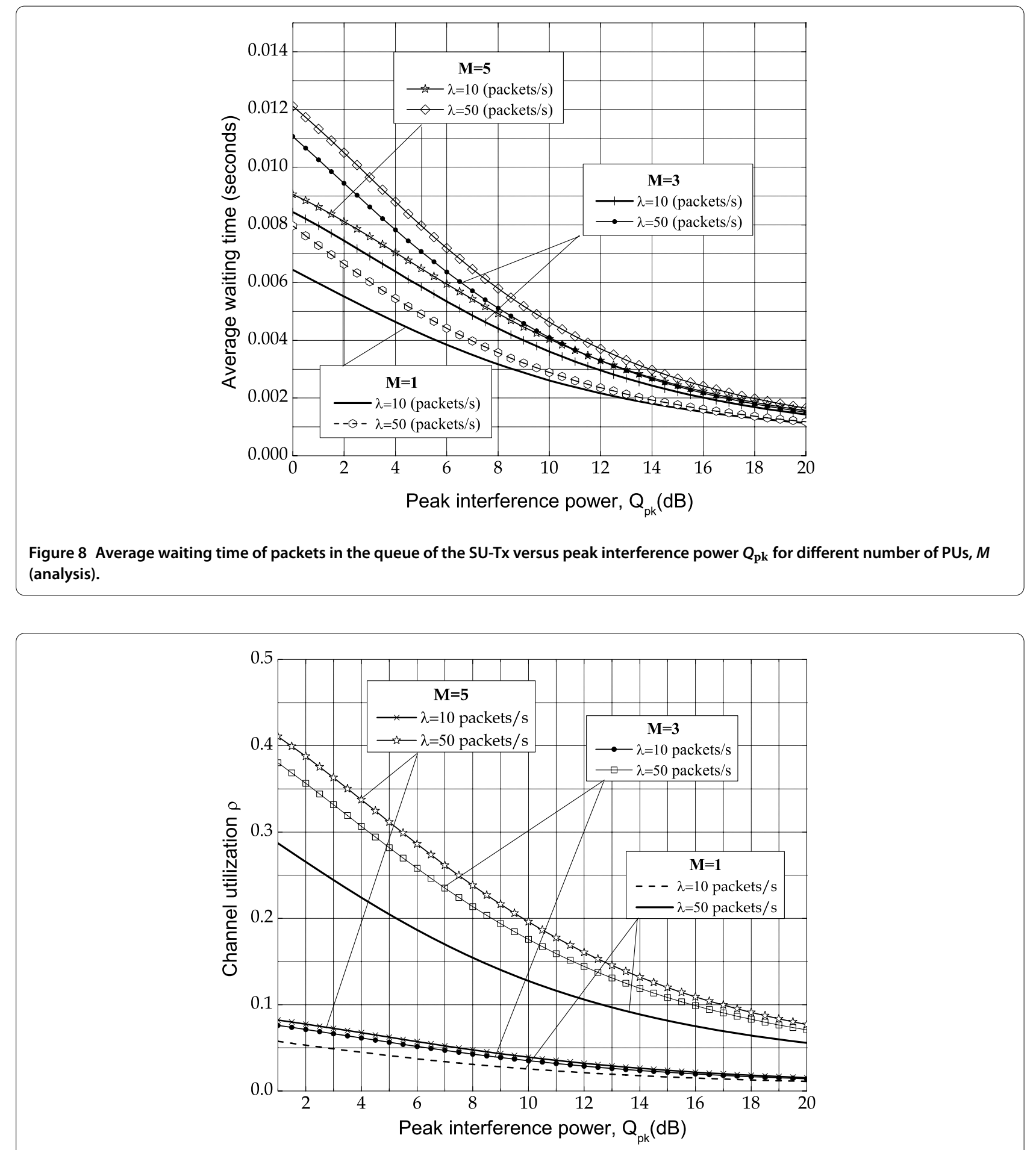

Figure 9 Impact of peak interference power $Q_{\mathrm{pk}}$ on the channel utilization $\rho$ with different number of PUs, $M$ (analysis).

tion for two values of average arrival rate given as $\lambda=$ 10,50 packets/s.

Figure 8 illustrates that the average waiting time increases as the number of PUs and arrival rate increase.
Apparently, these results are in line with the behavior observed for the outage probability and average transmission time above and may be explained as follows. At a fixed value of $Q_{\mathrm{pk}}$, an increasing number of PUs leads to an in- 


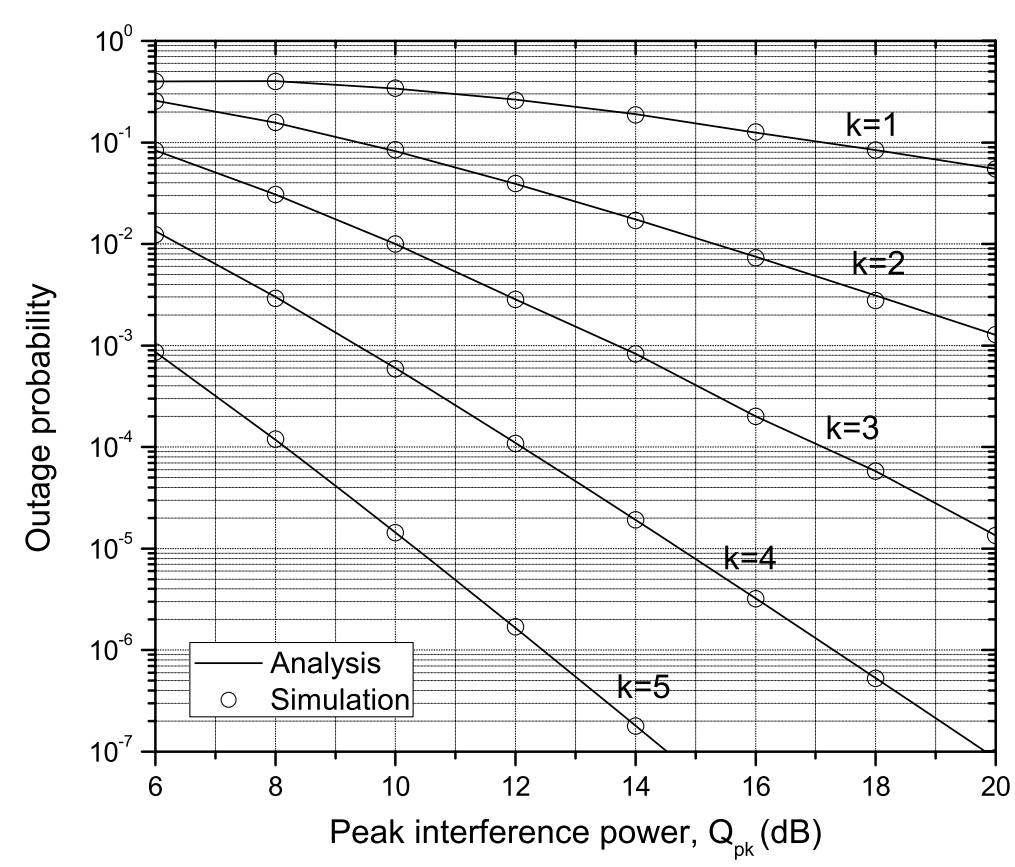

Figure 10 Impact of peak interference power $Q_{\mathrm{pk}}$ on the outage probability $P_{\text {out }}$ of point-to-multipoint communications in which the number of primary users and secondary users are set to $N=M=5$ ( $k$ denotes the number of secondary users that cannot receive the common packets successfully).

crease of average transmission time due to the reasons explained above and hence an increase of average waiting time. Similarly, when the arrival rate increases, the number of packets to be stored in the buffer increases as well and await transmission. On the other hand, as the transmission rate is restricted due to the peak interference power constraint, the packets have to stay longer in the buffer before they are transmitted.

Figure 9 provides insights into the stable transmission condition as a function of the peak interference power, $Q_{\mathrm{pk}}$, with the number of PUs given as $M=1,3,5$ and arrival rates being $\lambda=10,50$ packets/s. The results show that for a given value of the number of PUs, $M$, and fixed value of the peak interference power $Q_{\mathrm{pk}}$, the channel utilization $\rho=\lambda \mathbb{E}[T]$ for arrival rate $\lambda=10$ packets/s outperforms the result for $\lambda=50$ packets $/ \mathrm{s}$. In other words, the significant lower channel utilization for $\lambda=10$ packets/s compared to $\lambda=50$ packets $/ \mathrm{s}$ provides a more stable transmission with respect to the service rate $\mu$ in terms of the stable condition formulated in (36). Clearly, the service rate $\mu$ of an SU-Tx is restricted for a fixed value of the peak interference power $Q_{\mathrm{pk}}$ while a higher arrival rate causes more packets to be processed by the buffer expecting timely transmission. Accordingly, the ratio of arrival rate to service rate, relating to the stable transmission condition $\lambda / \mu<1$, has to be carefully considered in order to not exceed the capacity of the secondary system. It can also be observed from the figure that the stable transmission condition can be easily satisfied in the high regime of the peak interference power, say $Q_{\mathrm{pk}} \geq 16.5 \mathrm{~dB}$, as the channel utilization is sufficiently low.

\subsection{Point-to-multipoint communications}

We now focus on the impact of the peak interference power on the outage probability of the point-to-multipoint communications as shown in Figure 10. In particular, we set the number of SUs and PUs as $N=M=5$ and plot the outage probability as a function of the peak interference power, $Q_{\mathrm{pk}}$, under the condition that $k=1,2,3,4,5$ out of the total of $N=5 \mathrm{SU}-\mathrm{Rx}$ cannot receive the common packets successfully. Clearly, we can deduce from the results that the probability of exactly $k$ out of the $N=5$ SU-Rx not being able to receive the common packet successfully decreases as $k$ increases. In addition, the outage performance improves as the peak interference power increases as expected.

Figure 11 presents the probability that all SU-Rx can receive the common packets successfully as a function of the peak interference power for the number of PUs fixed to $M=8$ and the number of SUs given as $N=3,5,8$. This scenario relates to the best channel condition as outlined in Section 4.2. It can be seen from the figure that the probability of the SBS transmitting the common packets successfully to all SUs is quite high (above 0.8) in the high regime of the peak interference power, $Q_{\mathrm{pk}} \geq 19 \mathrm{~dB}$. The 


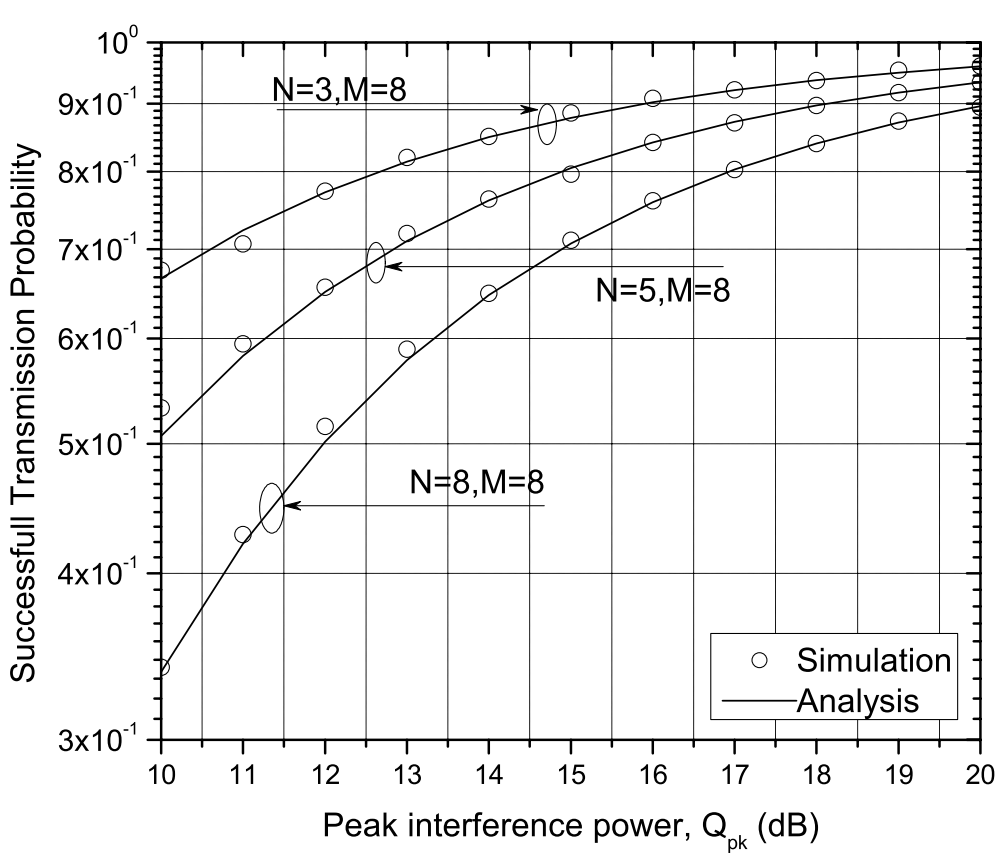

Figure 11 Impact of the peak interference power $Q_{\mathrm{pk}}$ on the probability of successful packet transmission for the best channel condition (number of PU-Rx, $M=8$; number of SU-Rx, $N=3,5,8$ ).

figure also indicates that for number of PUs fixed at $M=8$, the probability of successful transmission decreases with an increase of the number of SU-Rx, $N=3,5,8$. Similar to point-to-point communications, an increasing number of SUs leads to an increase of the peak interference power constraint at the SBS. Thus, the time it takes to transmit the common packet may be longer while the probability of successful transmission decrease for the best channel condition.

\section{Conclusions}

In this paper, we have analyzed the delay performance of spectrum sharing systems for point-to-point and pointto-multipoint communications. In particular, we have assumed that each packet has a delay threshold, transmission channels undergo Rayleigh fading, SUs posses perfect CSIs and ACKs are transmitted without error and delay. Closed-form expressions for the outage probability and average transmission time for point-to-point communications are obtained. In addition, we have utilized the M/G/1 queuing model to analyze the queueing characteristics of such systems including the average transmission time, the packet waiting time and the stable transmission condition of an SU. Based on the analytical framework established for point-to-point communications, we have also derived closed-form expressions for the outage probability and the successful transmission probability for point-to-multipoint communications under best channel conditions. Numerical results for representative scenarios have been provided to quantify the impact of an increase of the number of SUs and PUs on system performance. In particular, it has been shown that an increasing number of SUs or PUs significantly increases packet delay if the peak interference power is constraint by the PUs to be low while small performance degradation is observed if the PUs tolerate sufficiently large peak interference power. Accordingly, the developed analytical framework for point-to-point and point-to-multipoint communications in spectral sharing systems may serve to efficiently examine system performance. For example, it may be used to deduce a trade-off between QoS requirements of the secondary system and interference constraints posed by the primary system.

\section{Competing interests}

The authors declare that they have no competing interests.

\section{Acknowledgement}

Part of this work was presented at the IEEE International Symposium on Wireless and Pervasive Computing, Hong Kong, China, February 2011.

\section{Received: 26 August 2011 Accepted: 10 November 2011}

\section{References}

1. Facilitating opportunities for flexible, efficient, and reliable spectrum use employing cognitive radio technologies, Technical Report 03-108, Federal Communications Commission (FCC), 2005

2. J Mitola, GQ Maguire, Cognitive radio: Making software radios more personal. IEEE Pers Commun 6(4), 13-18 (1999) 
3. A Ghasemi, ES Sousa, Fundamental limits of spectrum-sharing in fading environments. IEEE Trans Wirel Commun 6(2), 649-658 (2007)

4. L Musavian, S Aissa, Fundamental capacity limits of cognitive radio in fading environments with imperfect channel information. IEEE Trans Commun 57(11), 3472-3480 (2009)

5. HA Suraweera, PJ Smith, M Shafi, Capacity limits and performance analysis of cognitive radio with imperfect channel knowledge. IEEE Trans Veh Technol 59(2), 1811-1822 (2010)

6. PA Dmochowski, HA Suraweera, PJ Smith, M Shafi, Impact of channel knowledge on cognitive radio system capacity, in Proc. IEEE Vehicular Technology Conference, Ottawa, Canada, September 2010, pp. 1-5

7. L Musavian, S Aissa, Capacity and power allocation for spectrum-sharing communications in fading channels. IEEE Trans Wirel Commun 8(1), 148-156 (2009)

8. D Wu, R Negi, Effective capacity: A wireless link model for support of quality of service. IEEE Trans Wirel Commun 2(4), 630-643 (2003)

9. L Musavian, S Aissa, Effective capacity of delay-constrained cognitive radio in Nakagami fading channels. IEEE Trans Wirel Commun 9(3), 1054-1062 (2010)

10. L Musavian, S Aissa, Cross-layer analysis of cognitive radio relay networks under quality of service constraints, in Proc. IEEE Vehicular Technology Conference, Barcelona, Spain, June 2009, pp. 1-5

11. H Tran, TQ Duong, H-J Zepernick, Queuing analysis for cognitive radio networks under peak interference power constraint, in Proc. IEEE International Symposium on Wireless and Pervasive Computing, Hong Kong, China, February 2011, pp. 1-5

12. H Tran, TQ Duong, H-J Zepernick, Performance of a spectrum sharing system over Nakagami-m fading channels, in Proc. International Conference on Signal Processing and Communication Systems, Gold Coast, Australia, December 2010, pp. 1-6

13. S Mangold, A Jarosch, C Monney, Operator assisted cognitive radio and dynamic spectrum assignment with dual beacons - detailed evaluation, in Proc. International Conference on Communication System Software and Middleware, New Delhi, India, August 2006, pp. 1-6

14. TW Ban, W Choi, BC Jung, DK Sung, Multi-user diversity in a spectrum sharing system. IEEE Trans Wirel Commun 8(1), 102-106 (2009)

15. X Kang, Y-C Liang, A Nallanathan, H Garg, R Zhang, Optimal power allocation for fading channels in cognitive radio networks: Ergodic capacity and outage capacity. IEEE Trans Wirel Commun 8(2), 940-950 (2009)

16. NB Mehta, V Sharma, G Bansal, Performance analysis of a cooperative system with rateless codes and buffered relays. IEEE Trans Wirel Commun 10(4), 1069-1081 (2011)

17. W Song, Y Cheng, W Zhuang, Improving voice and data services in cellular/WLAN integrated networks by admission control. IEEE Trans Wirel Commun 6(11), 4025-4037 (2007)

18. J Burdin, R Landry, Delay analysis of wireless Nakagami fading channels, in Proc. IEEE Global Telecommunications Conference, New Orleans, U.S.A., December 2008, pp. 1-5

19. N Ahmed, M Khojestapour, R Baraniuk, Delay-limited throughput maximization for fading channels using rate and power control, in Proc. IEEE Global Telecommunications Conference, Dallas, U.S.A., November 2004, pp. 3459-3463

20. SM Ross, Introduction to Probability Models, 9th edn. (Elsevier, Amsterdam, 2007)

21. ND Sidiropoulos, TN Davidson, ZQ Luo, Transmit beamforming for physical-layer multicasting. IEEE Trans Signal Process 54(6), 2239-2251 (2006)

22. N Jindal, ZQ Luo, Capacity limits of multiple antenna multicast, in Proc. IEEE International Symposium on Information Theory, July 2006, pp. 1841-1845

23. HA Suraweera, I Gao, PJ Smith, M Shafi, M Faulkner, Channel capacity limits of cognitive radio in asymmetric fading environments, in Proc. IEEE International Conference on Communications, Beijing, China, May 2008, pp. $4048-4053$

\section{doi:10.1186/1687-1499-2012-9}

Cite this article as: Tran et al.: Delay performance of cognitive radio networks for point-to-point and point-to-multipoint communications. EURASIP Journal on Wireless Communications and Networking 2012 2012:9.

\section{Submit your manuscript to a SpringerOpen ${ }^{\circ}$ journal and benefit from:}

Convenient online submission

- Rigorous peer review

- Immediate publication on acceptance

- Open access: articles freely available online

- High visibility within the field

- Retaining the copyright to your article

Submit your next manuscript at $\boldsymbol{\triangleright}$ springeropen.com 